\title{
Adiposidad visceral y resistencia insulínica: rol de la AMPK
}

Vega Joubert, M. • Oliva, M.E. • Ferreira, M.R. • D’Alessandro, M.E. 


\title{
ADIPOSIDAD VISCERAL Y RESISTENCIA INSULÍNICA: ROL DE LA AMPK
}

\author{
Vega Joubert, M. ${ }^{1}$, Oliva, M.E. ${ }^{1,2}$, Ferreira, M.R. ${ }^{1,2}$, D'Alessandro, M.E. ${ }^{1,2}$ \\ e-mail: medaless@fbcb.unl.edu.ar \\ ${ }^{1}$ Laboratorio de Estudio de Enfermedades Metabólicas Relacionadas con la Nutrición, Facultad de Bioquímica y Ciencias Biológicas, \\ Universidad Nacional del Litoral, Santa Fe, Argentina \\ ${ }^{2}$ Consejo Nacional de Investigaciones Científicas y Técnicas (CONICET), Ciudad Autónoma de Buenos Aires, Argentina
}

\section{RESUMEN}

Introducción: La disfunción del tejido adiposo constituye un punto central en el desarrollo de la resistencia insulínica y otras alteraciones asociadas al Síndrome Metabólico. La proteína quinasa activada por AMP (AMPK) es considerada un sensor metabólico celular, con un importante rol en la regulación de la actividad metabólica del tejido adiposo blanco.

Objetivos: Evaluar los efectos de la administración crónica de una dieta rica en sacarosa a ratas normales sobre algunos mecanismos involucrados en la disfunción del tejido adiposo y su relación con la resistencia insulínica.

Materiales y métodos: Ratas macho Wistar recibieron una dieta de referencia (DR) o una dieta rica en sacarosa (DRS) durante seis meses. Al final del período experimental se analizaron parámetros antropométricos e índice de adiposidad visceral. En tejido adiposo epididimal se midió: contenido de triglicéridos, actividad enzimática carnitina palmitoil transferasa (CPT1, CPT2 y CPTtotal) y masa proteica de la AMPK y AMPK fosforilada (AMPKp). Se determinaron los niveles séricos de triglicéridos, ácidos grasos no esterificados, glucosa, insulina y TNFa. La sensibilidad insulínica se midió por la técnica clamp euglucémica-hiperinsulinémica.

Resultados: Los animales dislipémicos insulinoresistentes alimentados con DRS presentaron un aumento significativo en los parámetros antropométricos (peso corporal, circunferencias toráxica y abdominal e indíce de masa corporal), y adiposidad visceral. En el tejido adiposo epididimal, se observó un aumento en el contenido de triglicéridos, sin modificaciones en el transporte de ácidos grasos hacia la mitocondria (estimado por la medida de la actividad CPT). Además, se observó una disminución en la masa proteica de la AMPKp que se correlacionó negativamente con los niveles séricos de TNFa. 
Conclusiones: Los hallazgos aportan nuevos mecanismos que involucran a la AMPK en la disfunción del tejido adiposo visceral en ratas dislipémicas insulinorresistentes.

Palabras clave: dieta rica en sacarosa, adiposidad visceral, resistencia insulínica, AMPK.

\section{SUMMARY}

Introduction: Adipose tissue dysfunction is a central point in the development of insulin resistance and other Metabolic Syndrome associated disorders. AMP-activated protein kinase (AMPK) is a cellular metabolic sensor, that has a key role in the regulation of white adipose tissue metabolic activity.

Objectives: This study evaluates the effects of a sucrose-rich diet chronic administration on some mechanisms involved in adipose tissue dysfunction and its relationship with insulin resistance.

Materials and methods: Male Wistar rats received a reference diet (RD) or a sucrose-rich diet (SRD) for six months. At the end of the experimental period, the anthropometric parameters and visceral adiposity index were analyzed. In epididymal adipose tissue: triglyceride content, carnitine palmitoyl transferase enzymes activities (CPT1, CPT2 and total CPT) and protein mass levels of AMPK and PAMPK were evaluated. Triglycerides, free fatty acid, glucose, insulin and TNFa serum levels were determined. Insulin sensitivity was measured by the euglycemic-hyperinsulinemic clamp technique.

Results: Insulin resistant and dyslipemic SRD-fed animals showed a significant increase in the anthropometric parameters (body weight, thoracic and abdominal circumferences, body mass index), and visceral adiposity index. In the epididymal adipose tissue, an increase in the triglyceride content was observed, without changes in mitocondrial transport of fatty acids (estimated by the CPT activity measurement). In addition, a decrease in the protein mass of the-pAMPK was observed, which was negatively correlated with TNF $\alpha$ serum levels.

Conclusions: The findings provide new mechanisms that involve AMPK in visceral adipose tissue dysfunction in insulin resistant dyslipemic rats.

Key words: sucrose-rich diet, visceral adiposity, insulin resistance, AMPK

\section{INTRODUCCIÓN}

El Síndrome Metabólico (SM) se define como un conjunto de desórdenes metabólicos interrelacionados cuyos componentes comprenden dislipemia, intolerancia a la glucosa, resistencia insulínica (RI), adiposidad visceral e hipertensión arterial acompañado de un estado protrombótico, proinflamatorio, prooxidante y esteatosis hepática no alcohólica, los cuales son factores de riesgo de Enfermedad Cardiovascular (ECV) y Diabetes tipo 2, patologías asociadas a elevadas tasas de morbi-mortalidad (1,2). Si bien el desarrollo del SM se encuentra asociado en parte a factores genéticos, la elevada prevalencia está ligada al estilo de vida actual de la población 
caracterizado por reducida actividad física, sedentarismo e incremento en la incorporación de nutrientes ricos en azúcares simples (fructosa/sacarosa), grasas saturadas y ácidos grasos poliinsaturados n-6 (n-6 PUFA) sumado esto a una disminución en la ingesta de proteínas, fibras y ácidos grasos poliinsaturados n-3 (n-3 PUFA) (3).

El SM constituye actualmente un importante problema desde el punto de vista de la Salud Pública y su prevalencia a nivel mundial es del $20 \%$ a 30\% en la población adulta (4). En Argentina, las cifras de prevalencia alcanzan el $27,5 \%$ (5). Por tal motivo resulta necesario dilucidar su fisiopatología, actualmente no esclarecida completamente, a fin de establecer estrategias terapéuticas tendientes a reducir su elevada prevalencia.

Se ha demostrado que la disfunción del tejido adiposo (TA) constituye un punto central en el desarrollo de las alteraciones asociadas al SM. El depósito visceral de la grasa, la hipertrofia y el cambio del perfil secretor de los adipocitos, junto con la infiltración del tejido adiposo por células inflamatorias son algunas de las características que determinan una comunicación alterada del TA con otros órganos. En relación con esto, la disfunción del TA, explicaría parte de la etiopatogenia de las enfermedades metabólicas y cardiovasculares asociadas a la obesidad (6). El TA es capaz de secretar diversas sustancias conocidas como adipoquinas que se encuentran implicadas en la regulación del peso corporal, en el sistema inmune, en la función vascular y en la sensibilidad insulínica (7).

La proteína quinasa activada por AMP (AMPK) participa en el mantenimiento de la homeostasis celular y la energía corporal en tejidos como el hígado y músculo. Cuando se activa (fosforilación), inhibe los procesos que consumen energía (lipogénesis, síntesis proteica, gluconeogénesis) y activa procesos que producen energía (oxidación de ácidos grasos). El TA es un órgano importante en la homeostasis energética y en la regulación de la sensibilidad a la insulina a través de la liberación de ácidos grasos y la secreción de hormonas. En los adipocitos, la activación de la AMPK puede conducir a una inhibición de la síntesis de ácidos grasos y una activación de la oxidación de los mismos. Además, la AMPK también inhibe la lipólisis. Todas estas acciones podrían ser beneficiosas en los estados de resistencia a la insulina, especialmente porque la activación de la AMPK también reduce la secreción de citoquinas inflamatorias en los adipocitos (8-10).

Trabajos previos del grupo, observaron que ratas alimentadas crónicamente con una dieta rica en sacarosa (DRS) (60-65\% de la energía total) presentan RI, dislipemia, acúmulo ectópico de lípidos (músculo esquelético/cardíaco, hígado y páncreas) y adiposidad visceral, por lo que representa un modelo experimental que mimetiza el fenotipo del SM humano $(11,12)$. Estos animales presentan un aumento de la masa del TA visceral epididimal y del tamaño de sus células adiposas (hipertrofia), acompañado de un aumento moderado en la lipólisis basal y una disminución en la acción antilipolítica de la insulina (13). Sin embargo, los posibles mecanismos moleculares implicados en la desregulación del TA en este modelo experimental y su relación con 
la RI no han sido completamente esclarecidos. Por lo tanto, el objetivo del presente estudio fue evaluar algunos mecanismos subyacentes que podrían estar involucrados en la disfunción del TA y RI de ratas alimentadas crónicamente con una dieta rica en sacarosa.

\section{MATERIALES Y MÉTODOS}

\section{Animales y dietas}

Se utilizaron ratas macho de la cepa Wistar provenientes del bioterio de la Facultad de Farmacia y Bioquímica de la Universidad de Buenos Aires (Buenos Aires, Argentina), con peso inicial aproximado de 180-200g. Desde su llegada, los animales se mantuvieron bajo condiciones controladas de luz-oscuridad de 12 hs (7:00 -19:00 hs), temperatura ( $22 \pm 1 \stackrel{\circ}{ } \mathrm{C}$ ), aireación y humedad ( $55 \pm 5 \%$ ), teniendo libre acceso al agua y a una dieta comercial de laboratorio conteniendo (\% en peso): carbohidratos (maíz, sorgo, trigo, avena, cebada) 42; proteínas 24; grasas 6; energía aportada 15,33 kJ/g (GEPSA FEED, Bs.As., Argentina). Luego de una semana de aclimatación, los animales se dividieron aleatoriamente en dos grupos: el grupo de referencia, continuó recibiendo la dieta comercial estándar para roedores (DR), el grupo experimental consumió una dieta semisintética rica en sacarosa (DRS) cuya composición estuvo basada en las recomendaciones del Comité ad hoc del "American Institute of Nutrition" (AIN-93) (14). La misma contenía (\% en peso): sacarosa: 62,5; aceite de maíz: 7; proteínas: 18; energía aportada 16,30 kJ/g. Ambas dietas (DR y DRS) se administraron "ad libitum" por un período de 6 meses.

Durante todo el período experimental se determinó la ganancia de peso y la ingesta energética de las ratas pertenecientes a cada grupo dietario. Los animales fueron manipulados cumpliendo con las guías internacionales del cuidado y buen uso de animales de laboratorio. El protocolo experimental fue evaluado y aprobado por el Comité de Ética y Seguridad de la Investigación de la Facultad de Bioquímica y Ciencias Biológicas UNL, Santa Fe, Argentina.

\section{Métodos analíticos}

Finalizado el período experimental, el alimento fue removido al final del período de oscuridad y los animales ( $\mathrm{n}=6$ de cada grupo) fueron anestesiados con pentobarbital sódico $(60 \mathrm{mg} / \mathrm{kg}$ peso corporal) administrado por vía intraperitoneal. Se evaluaron parámetros antropométricos: circunferencia abdominal (CA1 y CA2), circunferencia torácica (CT) y longitud naso-anal e Índice de Masa Corporal (IMC) $(15,16)$. Posteriormente, se obtuvieron muestras de sangre de la vena cava inferior y se centrifugaron rápidamente. El suero obtenido fue utilizado inmediatamente o conservado a $-20{ }^{\circ} \mathrm{C}$ hasta su procesamiento. Los TA epididimal, mesentérico y retroperitoneal fueron extraídos y pesados para el cálculo del Índice de adiposidad visceral (IAV) (17). El TA 
epididimal fue congelado a la temperatura del $\mathrm{N}_{2}$ líquido para su posterior procesamiento, a excepción del utilizado inmediatamente para determinar el contenido de triglicéridos tisular.

Los niveles séricos de triglicéridos $(\mathrm{Tg})$ y glucosa fueron cuantificados con kits enzimáticos comerciales (Wiener Lab., Rosario, Argentina). Los ácidos grasos no esterificados (AGNE) se determinaron usando un kit comercial (Randox, Reino Unido) basado en el método enzimático colorimétrico acilCoa sintetasa/acilCoA oxidasa. Los niveles de insulina se determinaron según se describió en trabajos previos (18). Los niveles de TNFa en suero se midieron utilizando un kit comercial de ELISA (Thermo Scientific, Rockford, EE.UU.).

\section{Clamp euglucémica hiperinsulinémica}

En un grupo de animales pertenecientes a ambos grupos dietarios ( $n=6$ de cada grupo) y ayunados por 5 hs se midió la sensibilidad insulínica a través de la técnica clamp euglucémica hiperinsulinémica. Para ello, se les infundió una solución de insulina humana recombinante (Humulin R, Lilly, Indianápolis, EE.UU.) a 0,8 U/Kg peso corporal $\mathrm{x}$ h durante $120 \mathrm{~min}$. La glucemia se mantuvo a un nivel euglucémico (5,5-6,0 mM) mediante la infusión de glucosa a velocidad variable. La velocidad de infusión de glucosa (VIG): mg de glucosa infundida calculada durante los últimos 60 min de la prueba (estado estacionario) es una medida de la sensibilidad insulínica periférica global. Detalles de la metodología han sido descriptos en trabajos previos (19). Los resultados se expresaron como [mg glucosa/(kg x min)].

\section{Contenido de triglicéridos en tejido adiposo epididimal}

Porciones distales de cada epidídimo fueron extraídas rápidamente y lavadas en solución salina tibia ( $\mathrm{NaCl} 0,9$ $\mathrm{g} \%$ a $37^{\circ} \mathrm{C}$ ) para la determinación del contenido lipídico total utilizando metodología previamente descripta por el grupo (13).

\section{Actividad enzimática CPT1, CPT2 y CPTtotal}

Las actividades de las enzimas CPT1, CPT2 y CPTtotal se determinaron espectrofotométricamente según la metodología descripta por Ling y col. (20) con breves modificaciones, y utilizada en trabajos previos del grupo (21). El tejido congelado fue homogenizado a $4^{\circ} \mathrm{C}$ en buffer de homogenización conteniendo: HEPES $20 \mathrm{mM}$; $\mathrm{KCl} 140 \mathrm{mM}$; EDTA $10 \mathrm{mM} ; \mathrm{MgCl}_{2} 5 \mathrm{mM}\left(\mathrm{pH}\right.$ 7,4) y se centrifugó a 500 xg a $4^{\circ} \mathrm{C}$ durante $10 \mathrm{~min}$. El sobrenadante obtenido fue centrifugado a $4^{\circ} \mathrm{C}$ a $9000 \mathrm{xg}$ por $45 \mathrm{~min}$ y el pellet mitocondrial resultante fue resuspendido en buffer de homogenización. Para determinar la actividad CPT total, una alícuota del extracto mitocondrial obtenido se adicionó a un medio de reacción compuesto por: HEPES 20 mM (pH: 7,4); EGTA 1 mM; sacarosa 220 mM; KCl 40 mM; DTNB 0,1 mM; albúmina de suero bovino 1,3 mM y palmitoil-CoA 40 $\mu \mathrm{M}$. La reacción se inició 
con el agregado de L-carnitina (concentración final $1 \mathrm{mM}$ ). La formación de TNB-CoAS se monitoreó continuamente a $412 \mathrm{~nm}$ durante 5 minutos a $37^{\circ} \mathrm{C}$. La actividad CPT total se calculó en base a la variación de la absorbancia por minuto y al coeficiente de extinción molar del DTNB ( $\varepsilon=13,6 \mathrm{mM})$. La actividad de la CPT2 se determinó en las mismas condiciones de ensayo, en presencia de malonil-CoA $10 \mu \mathrm{M}$ (inhibidor de la CPT1) en el medio de reacción. La actividad CPT1 se calculó como la diferencia entre la CPT total y CPT2. Los resultados se expresaron como $\mathrm{mU} / \mathrm{mL}$.

\section{Niveles de masa proteica de AMPK y AMPKp}

Los niveles de masa proteica de AMPKp (Thr172) fueron determinados mediante la técnica de Western Blot en extractos proteicos de tejido adiposo obtenidos como se describió anteriormente en trabajos del grupo $(21,22)$. Las proteínas, cuantificadas mediante el ensayo de Bradford (SIGMA), se separaron mediante SDSPAGE y se transfirieron a membranas de PVDF. Para el inmunobloting las membranas se expusieron con anticuerpos primarios específicos: policlonal anti-AMPK o anti-AMPKp (Thr172) (Santa Cruz Biotechnology, Inc.). Luego se incubaron con el anticuerpo secundario conjugado con horseradish-peroxidasa (ISAL-FBCB-UNL) durante 2 horas, con agitación y a temperatura ambiente. Las bandas fueron visualizadas por quimioluminiscencia de acuerdo a las instrucciones del fabricante (Super Signal West Pico Chemiluminiscent Substrate, Pierce Biotechnology, Rockford, IL). La intensidad de las bandas se cuantificó utilizando el software para capturar imágenes Scion Image versión 4,0.

La relación entre la cantidad de muestra procesada en el inmunoblotting y la intensidad de señal observada fue lineal bajo las condiciones previamente descriptas. Los niveles de la masa proteica de la proteína en estudio fueron normalizados con los niveles de la masa proteica del marcador $\beta$ actina (Santa Cruz Biotechnology, Inc., Santa Cruz, CA, USA).

\section{Análisis estadístico}

Los resultados se expresan como media \pm SEM. Las comparaciones de los datos obtenidos fueron realizadas mediante estadística paramétrica o no paramétrica (Test $\mathrm{t}$, test de Mann Whitney), de acuerdo a las características de los datos, evaluando normalidad y homogeneidad de varianzas (Test de Levene y Test de ShapiroWilk). La correlación entre los niveles de masa proteica de AMPKp y los niveles séricos de TNFa se determinó utilizando el coeficiente de correlación de Pearson. Diferencias de $p<0,05$ fueron consideradas estadísticamente diferentes. Los tratamientos estadísticos se realizaron con el software SPSS 17,0 for Windows (SPSS INC. Chicago, Illinois) (23). 


\section{RESULTADOS}

\section{Metabolitos séricos y sensibilidad insulinica}

Los niveles séricos de Tg, AGNE y glucosa son significativamente más elevados $(p<0,05)$ (Figura 1 ) en los animales alimentados con DRS cuando se los compara con el grupo DR. Los niveles de insulina plasmática son similares entre los grupos dietarios. Además, los animales alimentados con DRS presentan una marcada disminución $(p<0,05)$ de la sensibilidad insulínica periférica global $(V I G)$ respecto al grupo DR.

\section{Parámetros antropométricos, índice de masa corporal e ingesta energética}

Al final del periodo experimental los animales alimentados con DRS presentan un significativo incremento en el peso corporal y la ingesta energética $(p<0,05)$ cuando se los compara con el grupo $D R$, lo que concuerda con trabajos previos del grupo $(17,18)$ (Tabla 1). Además, estos animales presentan un aumento significativo $(p<0,05)$ en CA1, CA2 y CT sin cambios en la longitud naso-anal en comparación con el grupo DR. También se observa un aumento significativo $(p<0,05)$ en el IMC en los animales alimentados con DRS en comparación con los animales alimentados con DR.

\section{Pesos de los tejidos adiposos e índice de adiposidad visceral}

Los animales alimentados con DRS presentan un aumento significativo $(p<0,05)$ en el peso de los TA epididimal, retroperitoneal y mesentérico, acompañado de un incremento significativo $(p<0,05)$ en el IAV comparado con los animales alimentados con DR (Figura 2).

\section{Contenido de triglicéridos celular medio y actividades enzimáticas de CPT1, CPT2 y CPTtotal en tejido adiposo epididimal}

El contenido de triglicéridos celular medio se encuentra significativamente aumentado en los animales alimentados con DRS en comparación con los animales del grupo DR. Los valores se expresan como (nmol/célula): DR: $0,30 \pm 0,02$; DRS: $0,59 \pm 0,04 *{ }^{*} p<0,05$ DRS vs DR. Las actividades CPT1, CPT2 y CPTtotal son similares entre los grupos experimentales (Tabla 2). 


\section{Masa proteica de AMPK y AMPKp en tejido adiposo epididimal y su correlación con TNFa.}

El análisis cuantitativo de los inmunoblots demuestra que la abundancia relativa de la AMPKtotal es similar entre ambos grupos experimentales (Figura 3a). Por su parte, la AMPKp se encuentra significativamente disminuida $(p<0,05)$ en los animales alimentados crónicamente con DRS respecto a los animales alimentados con DR. En la Figura 3b, se observa una correlación negativa entre los niveles séricos de TNFa y la masa proteica de AMPKp en TA epididimal. En el grupo DRS la disminución de la masa proteica de la AMPKp en TA se asocia con niveles aumentados de TNF $\alpha$ en suero.

\section{DISCUSIÓN}

Los resultados obtenidos aportaron nuevos hallazgos relacionados a la disfunción del TA visceral en el modelo experimental utilizado. Hasta el presente, hay poca información disponible sobre la relación entre obesidad y parámetros antropométricos en animales de laboratorio. En este sentido, Novellli y col. (15) observaron en ratas normales que recibieron sacarosa al $30 \%$ en agua de bebida y una dieta alta en carbohidratos, un aumento significativo en la ganancia de peso e ingesta energética, acompañado por un incremento en la circunferencia torácica, circunferencia abdominal e índice de masa corporal. En nuestro trabajo encontramos que los animales alimentados crónicamente con DRS presentaron un incremento significativo en el peso corporal e ingesta energética, junto con un aumento en CA1, CA2, CT e IMC.

El incremento en la adiposidad visceral es un componente central en el SM y se relaciona estrechamente con la RI. En esta línea, Della Vedova y col. (24) observaron en ratones C57BL/6J alimentados con dieta alta en grasa y fructosa que el incremento en el índice de adiposidad visceral se asoció con un deterioro de la sensibilidad insulínica. En concordancia con esos resultados, en el presente estudio y en trabajos previos del grupo $(17,18)$ se observó que los animales alimentados con DRS presentaron un aumento significativo en el IAV junto con una marcada disminución de la sensibilidad insulínica periférica global.

Trabajos previos de nuestro grupo (11) demostraron en animales alimentados crónicamente con DRS, una mayor disponibilidad de lípidos plasmáticos (Tg, AGNE) consecuencia de un incremento en la síntesis y secreción hepática de VLDL-Tg y menor remoción plasmática de los mismos. Además, el TA de estos animales presenta hipertrofia de los adipocitos con aumento de la lipolisis y menor acción antilipolítica de la insulina lo que conduce a una mayor liberación de ácidos grasos libres a la circulación $(13,17)$. Al respecto, Grundy (6) sugiere que una ingesta alta en carbohidratos induce una mayor lipólisis que se asocia a un aumento de la lipogénesis de novo y acúmulo de Tg en TA. En este sentido, en el presente trabajo se encontró un aumento del contenido de $\mathrm{Tg}$, lo que se ha demostrado en estudios previos (17) que se encuentra acompañado de un aumento de la lipogénesis de novo en TA epididimal de las ratas alimentadas crónicamente con DRS. 
Por otra parte, otro mecanismo posiblemente involucrado en el acúmulo de lípidos en el TA es una menor oxidación de ácidos grasos, estimada a través de la enzima CPT1. Actualmente, debido al bajo rendimiento mitocondrial del tejido adiposo blanco, son escasos los estudios que investigan la vía oxidativa de los ácidos grasos en adipocitos en condiciones de obesidad y diabetes (25). Gaidhu y col. (26) observaron en ratones machos C57BL/6J alimentados durante 8 semanas con dieta rica en grasa una disminución significativa de la capacidad oxidativa de los adipocitos, junto con una reducción significativa en la expresión génica de PGC-1 $\alpha$ y PPAR $\alpha$, reguladores críticos de la biogénesis mitocondrial y el gasto energético. Sin embargo, en el presente trabajo no se observaron diferencias significativas en las actividades enzimáticas de CPT1, CPT2 y CPTtotal entre los grupos experimentales. Por lo tanto, podemos sugerir que el acúmulo de triglicéridos y adiposidad visceral observado en nuestro modelo experimental podría deberse a un aumento en la vía lipogénica sin cambios en los parámetros de la vía oxidativa estudiados.

En los últimos años varios autores han propuesto, en estudios en humanos y animales, que la obesidad, Rl y diabetes tipo 2 están asociados a una reducción de la AMPK en TA. $(27,28)$. Lindholm y col. (29) observaron en ratas Sprague-Dawley insulinoresistentes alimentadas con dieta alta en grasa por 18 semanas, una disminución en la actividad de la AMPK en tejido adiposo blanco. En esta línea, Gaidhu y col. (26) demostraron en ratones machos $\mathrm{C} 57 \mathrm{BL} / 6 \mathrm{~J}$ alimentados durante 8 semanas con dieta rica en grasa una disminución significativa en la masa proteica de la AMPKp. En concordancia con estos resultados, hemos encontrado que los animales alimentados con DRS, presentan una disminuida masa proteica de la AMPKp en el TA epididimal comparado con los animales alimentados con dieta de referencia.

Por último, es conocido que en la obesidad y alteraciones asociadas al SM se observa que un aumento en el tamaño del TA está asociado estrechamente a un aumento en la liberación de citoquinas proinflamatorias tales como IL-6, IL-1 y TNFa. Más recientemente, se ha demostrado que la activación de AMPK en tejido adiposo reduce la secreción de citoquinas proinflamatorias, entre ellas el TNFa (30). Lonzetti Bargut y col. (31) observaron en tejido adiposo epididimal de ratones machos C57BL/6 alimentados durante 8 semanas con dieta alta en grasa una disminución en la expresión proteica de la AMPKp y un aumento significativo en los niveles de ARNm de TNFa. En esta línea, en nuestro trabajo pudimos observar una correlación negativa entre la AMPKp en TA y los niveles de TNFa séricos. Además, en trabajos previos del grupo (18) se observó un aumento significativo en el contenido de TNFa en el TA de ratas alimentadas con DRS por largo plazo. La inhibición de AMPK en el tejido adiposo podría favorecer el aumento de TNFa y contribuir al desarrollo de trastornos asociados con la adiposidad visceral y la resistencia a la insulina. 


\section{CONCLUSIÓN}

Los resultados obtenidos en el presente trabajo aportan nuevos mecanismos que involucran a la AMPK en la disfunción del tejido adiposo en el modelo experimental utilizado por el grupo, el cual a través de la administración crónica de una dieta rica en sacarosa (DRS) mimetiza varios aspectos del Síndrome Metabólico humano.

\section{REFERENCIAS BIBLIOGRÁFICAS}

1. Bruce, K.; Hanson, M. 2010. The developmental origins, mechanisms, and implications of metabolic syndrome. J Nutr 140, 3: 648-652.

2. O'Neill, S.; O'Driscoll, L. 2015. Metabolic syndrome: a closer look th the growing epidemic and its associated pathologies. Obesity reviews 16: 1-12.

3. Myles, I. A. (2014). Fast food fever: reviewing the impacts of the western diet on immunity. Nutrition Journal, 13,61 .

4. Grundy, SM. 2008. Metabolic Syndrome Pandemic. Arteriosclerosis, Thrombosis, and Vascular Biology 28: 629-636.

5. Diaz, A.; Espeche, W.; March, C. Flores, R.; Parodi, R.; Genesio, M. A.; Sabio, R.; Poppe, S. 2017. Prevalencia del síndrome metabólico en Argentina en los últimos 25 años: revisión sistemática de estudios observacionales poblacionales. Hipertensión y riesgo vascular. Disponible en: http://dx.doi.org/10.1016/j.hipert.2017.08.003.

6. Grundy, SM. 2015. Adipose tissue and metabolic syndrome: too much, too little or neither. Eur J Clin Invest, 45 (11): 1209-1217.

7. Maury, E.; S.M.Brichard, S.M. 2010. Adipokine dysregulation, adipose tissue inflammation and metabolic syndrome. Molecular and Cellular Endocrinology 314: 1-16

8. Daval, M.; Foufelle,, F.; Ferre, P. (2006). Functions of AMP-activated protein kinase in adipose tissue. J. Physiol. 574: 55-62.

9. O'Neill, HM.; Holloway, GP.; Steinberg, GR. (2013). AMPK regulation of fatty acid metabolism and mitochondrial biogenesis: implications for obesity. Mol. Cell. Endocrinol. 366, 135-151.

10. Wu, L.; Zhang, L.; Bohan Li, Jiang, H.; Duan, Y.; Xie, Z.; Shuai, L.; Li, J.; Jingya Li, J.

Front Physiol. 2018. AMP-Activated Protein Kinase (AMPK) Regulates Energy Metabolism through Modulating Thermogenesis in Adipose Tissue. Front Physiol. 9: 122.

11. Lombardo, YB.; Chicco, A. 2006. Effects of dietary polyunsaturated n-3 fatty acids on dyslipidemia and insulin resistance in rodents and humans. A review. J Nutr Biochem 17:1-13. 
12. Rossi, AS.; Lombardo, YB.; Lacorte, JM.; Chicco, AG.; Rouault, C.; Slama, G.; Rizkalla, SW. 2005. Dietary fish oil positively regulates plasma leptin and adiponectin levels in sucrose-fed, insulin-resistant rats. Am J Physiol Regul Integr Comp Physiol 289: R486-R494.

13. Soria, A.; D'Alessandro, ME.; Lombardo, YB. 2001. Duration of feeding on a sucrose-rich diet determines metabolic and morphological changes in rat adipocytes. J Appl Physiol 91: 2109-2116.

14. Reeves, Nielsen F.H.; Fahey, G.C. 1993. AIN-93 Purified Diets for Laboratory Rodents: Final Report of the American Institute of Nutrition Ad Hoc Writing Committee on the Reformulation of the AIN-76A Rodent Diet. J. Nutr. 123, 11: 1939-1951.

15. Novelli, EL.; Diniz, YS.; Galhardi CM.; Ebaid, GM.; Rodrigues HG.; Mani, F.; Fernandes AA.; Cicogna AC.; Novelli Filho, JL. 2007. Anthropometrical parameters and markers of obesity in rats Laboratory Animals 41: 111-119.

16. Cossio Bolanos, MA.; Gomez Campos, R.; De Arruda, M.; Tadep, R.; Franssen-van, HN. 2010. Reliability values of somatic indicators in male wistar rats. Epidemiología e Investigación Básica 11, 296-302.

17. Rossi, AS; Oliva, ME.; Ferreira, MR.; Lombardo, YB.; Chicco, A. 2013. Dietary chia seed induced changes in hepatic transcription factors andtheir target lipogenic and oxidative enzyme activities in dyslipidaemic insulin-resistant rat. Br J Nutr. 109 (9): 1617-1627.

18. D’Alessandro, M.E.; Selenscig, D.; Illesca, P.; Chicco, A.; Lombardo, Y.B. 2015. Time course of adipose tissue dysfunction associated with antioxidant defense, inflammatory cytokines and oxidative stress in dyslipemic insulin resistant rats. Food Funct. 6, 4: 1299-1309.

19. D’Alessandro, ME.; Chicco, A.; Karabatas. L.; Lombardo, YB. 2000. Role of skeletal muscle on impaired insulin sensitivity in rats fed a sucrose rich diet: effect of moderate levels of dietary fish oil. J Nutr Biochem; 11(5): 273-80.

20. Ling, B.; Aziz, C.; Alcorn, J. 2012. Systematic evaluation of key L-Carnitine homeostasis mechanisms during postnatal development in rat. Nutrition \& Metabolism 9: 66.

21. Creus, A.; Ferreira, M.; Oliva, M.; Lombardo, YB. 2016. Mechanisms involved in the improvement of lipotoxicity and impaired lipid metabolism by dietary $\alpha$-linolenic acid rich salvia hispanica L (Salba) seed in the heart of dyslipemic insulin-resistant rats. J Clin Med. 28: 5(2).

22. Oliva ME, Creus A, Ferreira MR, Chicco, A.; Lombardo, YB. 2018. Dietary soya protein improves intramyocardial lipid deposition and altered glucose metabolism in a hypertensive, dyslipidaemic, insulinresistant rat model. Br J Nutr. 119(2): 131-42.

23. Snedecor, GWP.; Cochran, WG. 1967. "Statistical methods". Ames (lowa): lowa University Press; 339-350. 
24. Della Vedova, MC.; Muñoz, MD.; Santillan LD.; Plateo-Pignatari, MG.; Germanó, MJ.; Rinaldi Tosi, ME.; Garcia S.; Gomez, NN.; Fornes MW.; Gomez Mejiba SE.; Ramirez, D. 2016. Mouse Model of Diet-Induced Obesity Resembling Most Features of Human Metabolic Syndrome. Nutr Metab Insights 9: 93102.

25. Boudina, S; Graham, TE. 2014. Mitochondrial function/dysfunction in white adipose tissue. Exp Physiol 99:9 1168-1178.

26. Gaidhu, MP.; Anthony, NM.; Patel P.; Hawke, TJ.;Ceddia, RB. 2010. Dysregulation of lipolysis and lipid metabolism in visceral and subcutaneous adipocytes by high-fat diet: role of ATGL, HSL, and AMPK. Am J Physiol Cell Physiol 298: C961-C971.

27. Bijland, S.; Mancini SJ.; Salt IP. 2013. Role of AMP-activated protein kinase in adipose tissue metabolism and inflammation. Clinical Science 124, 491-507.

28. Mottillo EP.; Desjardins, EM.; Crane, JD.; Smith BK.; Green, AE.; Ducommun, S.; Henriksen, TI.; Rebalka, IA.; Razi, A.; Sakamoto, K.; Scheele, C.; Kemp, BE.; J. Hawke, TJ.; Ortega, J.; Granneman, JG.; Steinberg, GR. 2016. Lack of adipocyte AMPK exacerbates insulin resistance and hepatic steatosis through brown and beige Adipose tissue function. Cell Metab. 12, 24(1): 118-129.

29. Lindholm, CR.; Ertel, RL.; Bauwens, JD.; Schmuck, EG.; Jacob D. Mulligan, JD. 2013. A high-fat diet decreases AMPK activity in multiple tissues in the absence of hyperglycemia or systemic inflammation in rats. J Physiol Biochem 69(2): 165-175.

30. Mancini, SJ.; White, AD.; Bijland, S.; Rutherford, C.; Graham, D.; Richter, EA.; Viollet, B.; Rhian M. Touyz, RM.; Palmer, TM.; Salt, IP. 2017. Activation of AMP-activated protein kinase rapidly suppresses multiple pro-inflammatory pathways in adipocytes including IL-1 receptor-associated kinase-4 phosphorylation. Molecular and Cellular Endocrinology 440: 44-56.

31. Lonzetti Bargut, TC.; Mandarim de Lacerda, CA.; Barbosa Aguila, MB. 2015. A high-fish-oil diet prevents adiposity and modulates white adipose tissue inflammation pathways in mice. Journal of Nutritional Biochemistry 26, 960-969.

\section{Agradecimientos}

El presente estudio fue financiado por subsidios del Consejo Nacional de Investigaciones Científicas y Técnicas (CONICET) (Proyecto PIP \# 1122015 0100023CO) y Universidad Nacional del Litoral (CAI+D \# 50420150100011LI). Los autores agradecen a Silvia Rodríguez por su asistencia técnica. 

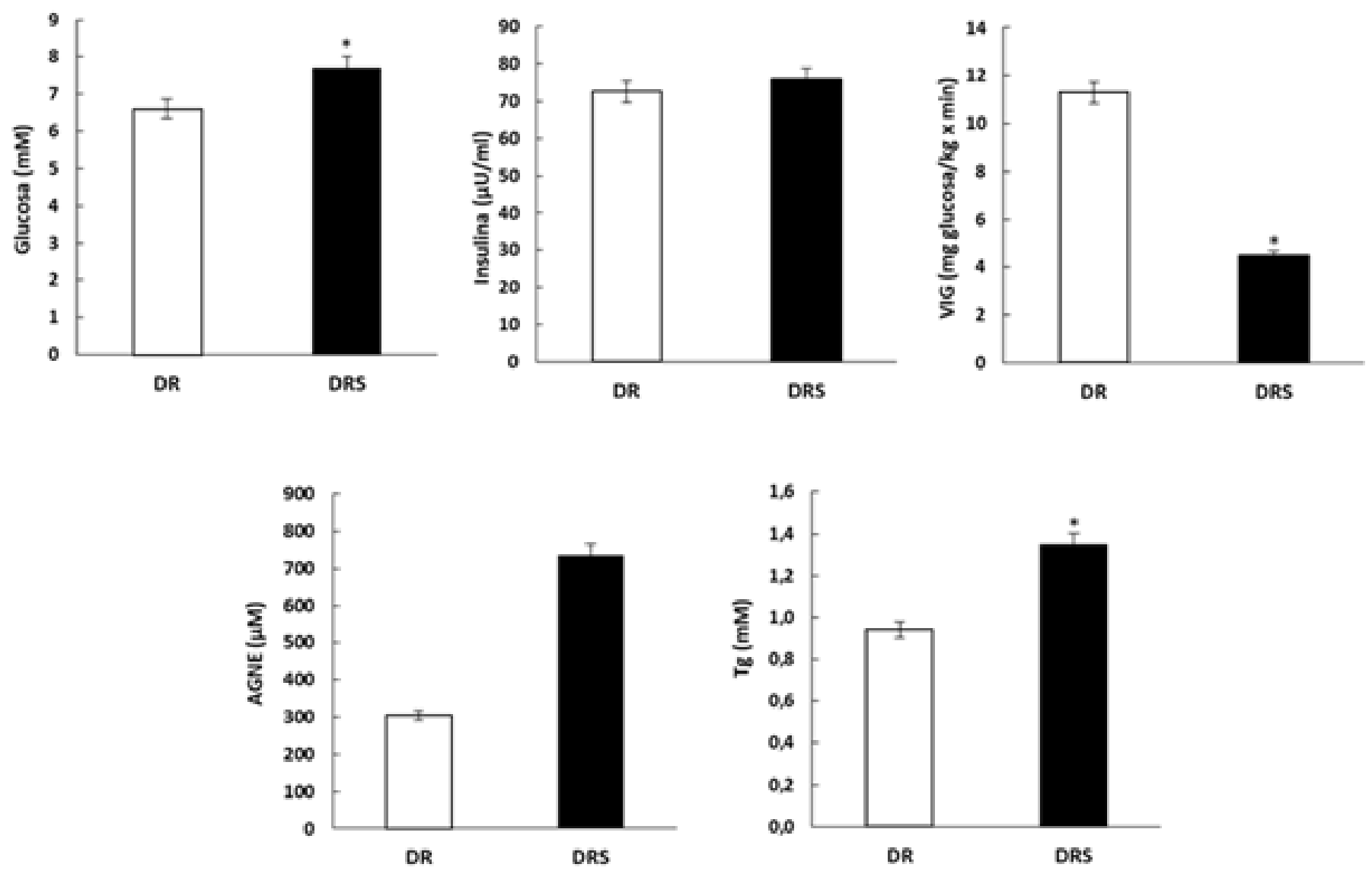

Figura 1. Metabolitos séricos y sensibilidad insulínica en los animales alimentados con dieta de referencia (DR) y dieta rica en sacarosa (DRS). Los resultados son expresados como media \pm SEM $(n=6) .{ }^{*} p<0,05$ DRS vs. DR. 

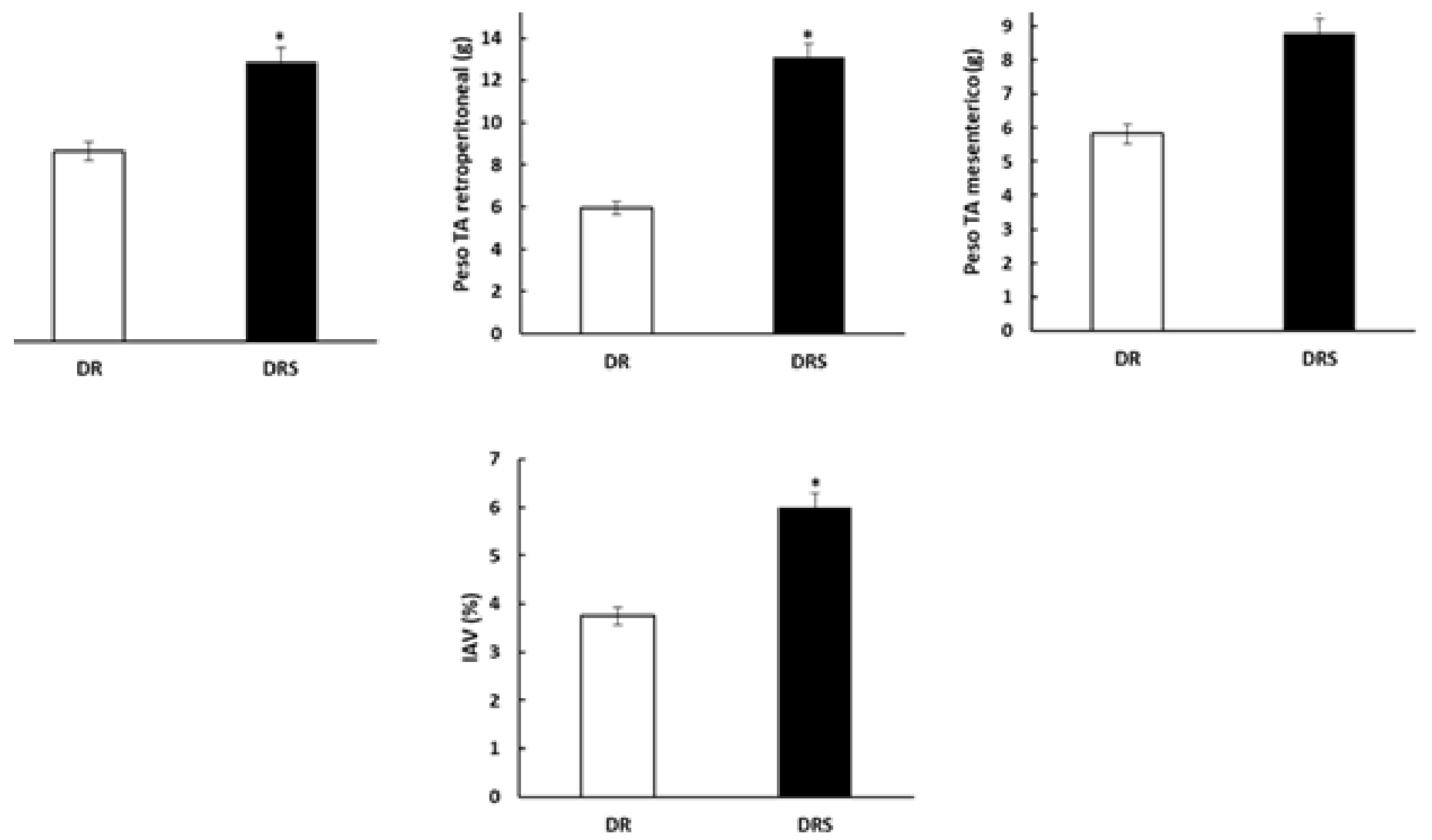

Figura 2. Peso de los tejidos adiposos epididimal, retroperitoneal y mesentérico e índice de adiposidad visceral en los animales alimentados con dieta de referencia (DR) y dieta rica en sacarosa (DRS). Los resultados son expresados como media \pm SEM ( $n=6)$. * $\mathrm{p}<0,05$ DRS vs. DR. 
a)

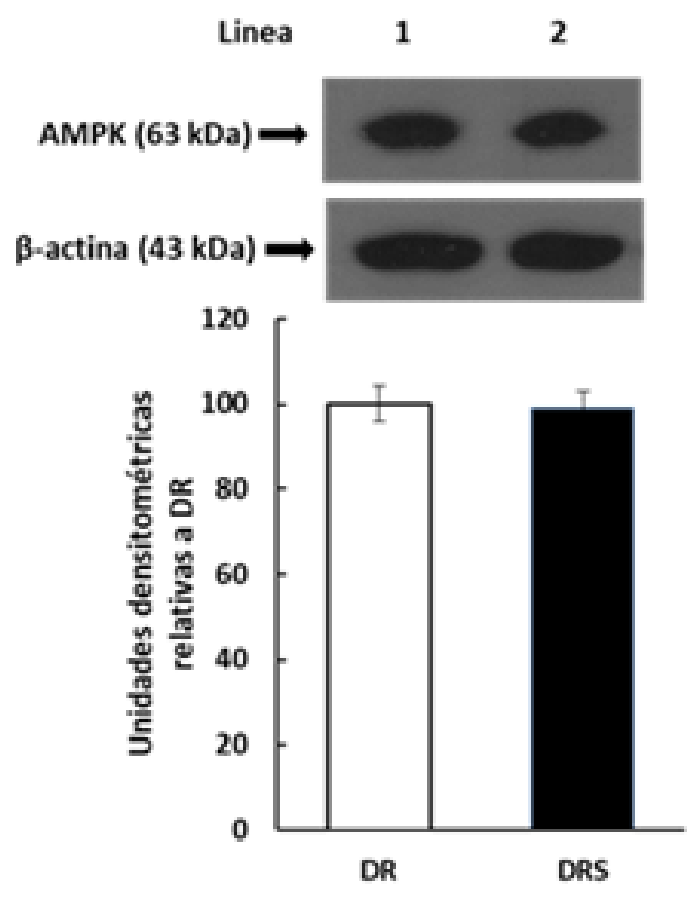

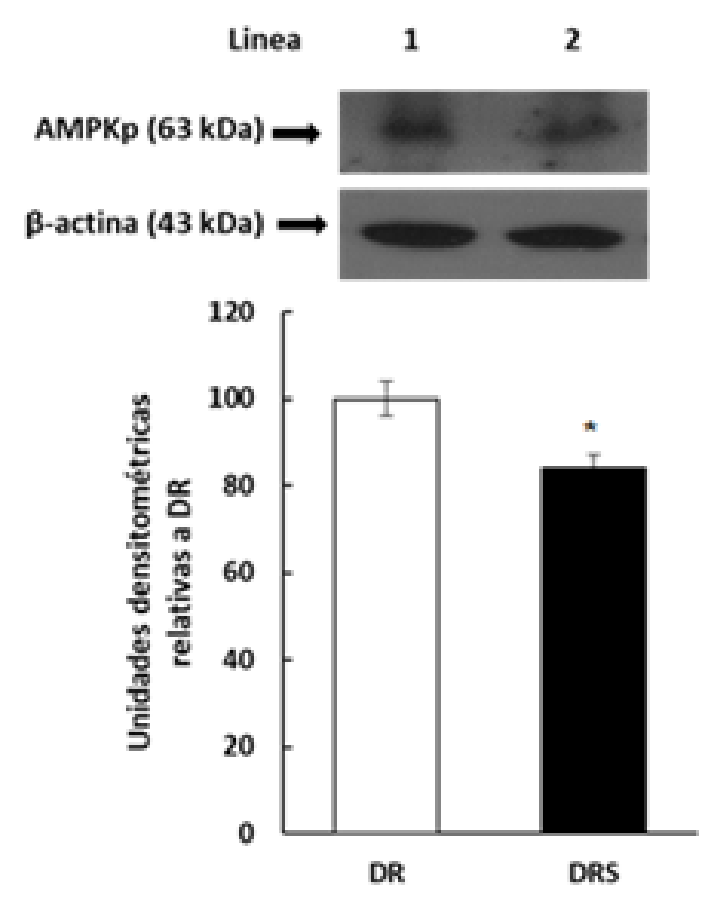

b)

AMPKp vs TNF $\alpha$

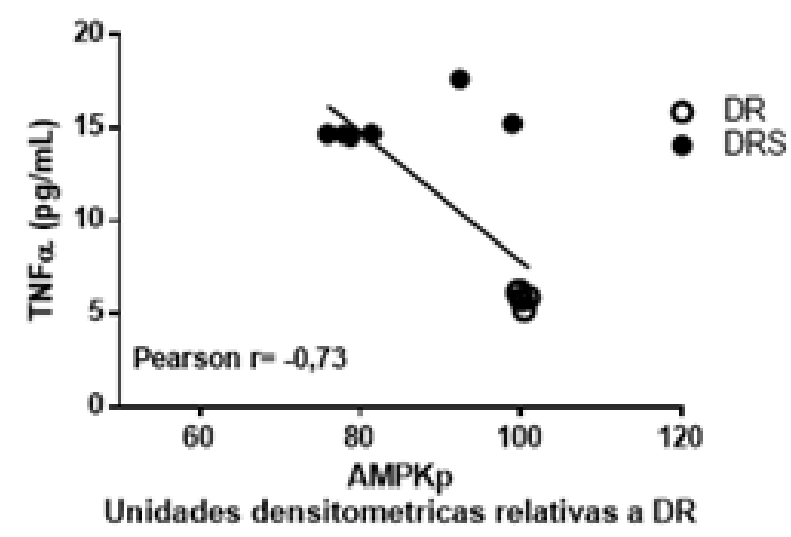

Figura 3. Masa proteica de AMPK y AMPKp en tejido adiposo epididimal y su correlación con los niveles de TNF $\alpha$ séricos en los animales alimentados con dieta de referencia (DR) y dieta rica en sacarosa (DRS). Los resultados son expresados como media \pm SEM $(n=6) .{ }^{*} p<0,05$ DRS vs. DR. 
Tabla 1. Parámetros antropométricos, índice de masa corporal e ingesta calórica en los animales alimentados con dieta de referencia (DR) y dieta rica en sacarosa (DRS).

\begin{tabular}{|l|c|c|}
\hline Parámetros antropométricos & DR & DRS \\
\hline Peso corporal Inicial (g) & $195,7 \pm 3,0^{\mathrm{a}}$ & $194,8 \pm 2,3^{\mathrm{a}}$ \\
\hline Peso corporal Final (g) & $501,8 \pm 8,5^{\mathrm{b}}$ & $545,7 \pm 5,6^{\mathrm{a}}$ \\
\hline CA1 $^{\mathbf{1}}$ (cm) & $21,2 \pm 0,2^{\mathrm{b}}$ & $23,3 \pm 0,6^{\mathrm{a}}$ \\
\hline CA2 $^{\mathbf{2}}$ (cm) & $21,1 \pm 0,2^{\mathrm{b}}$ & $23,4 \pm 0,5^{\mathrm{a}}$ \\
\hline CT (cm) $^{\mathbf{c}}$ & $19,1 \pm 0,2^{\mathrm{b}}$ & $21,5 \pm 0,4^{\mathrm{a}}$ \\
\hline Longitud Naso-anal (cm) $^{\mathbf{2}}$ & $26,3 \pm 0,3^{\mathrm{a}}$ & $25,9 \pm 0,2^{\mathrm{a}}$ \\
\hline Índices Somáticos & & $0,88 \pm 0,03^{\mathrm{a}}$ \\
\hline IMC (g/cm ) & $0,78 \pm 0,02^{\mathrm{b}}$ & \\
\hline Ingesta energética & $296,3 \pm 11,3^{\mathrm{a}}$ & $290,3 \pm 7,3^{\mathrm{a}}$ \\
\hline Inicial (kJ/día) & $298,4 \pm 10,3^{\mathrm{b}}$ & $386,5 \pm 15,8^{\mathrm{a}}$ \\
\hline Final (kJ/día) & & \\
\hline
\end{tabular}

Los valores se expresan como media \pm SEM. Los valores de cada fila que no comparten la misma letra son estadísticamente diferentes $(p<0,05)$. ${ }^{1-C A} 1$ es la medición de la circunferencia abdominal inmediatamente delante de las patas traseras. ${ }^{2-C A 2}$ es la medición de la circunferencia abdominal en la parte más ancha del abdomen.

Tabla 2. Actividades enzimáticas CPT1, CPT2 y CPT total en tejido adiposo epididimal de animales alimentados con dieta de referencia (DR) y dieta rica en sacarosa (DRS).

\begin{tabular}{|c|c|c|}
\hline & DR & DRS \\
\hline CPT1 $(\mathrm{mU} / \mathrm{mL})$ & $1,48 \pm 0,17^{\mathrm{a}}$ & $2,06 \pm 0,32^{\mathrm{a}}$ \\
\hline $\mathrm{CPT} 2(\mathrm{mU} / \mathrm{mL})$ & $2,84 \pm 0,16^{\mathrm{a}}$ & $2,87 \pm 0,15^{\mathrm{a}}$ \\
\hline CPTtotal $(\mathrm{mU} / \mathrm{mL})$ & $4,31 \pm 0,16^{\mathrm{a}}$ & $4,93 \pm 0,24^{\mathrm{a}}$ \\
\hline
\end{tabular}

Los valores se expresan como media \pm SEM. Los valores de cada fila que no comparten la misma letra son estadísticamente diferentes $(p<0,05)$. 